\title{
New Zealand White Rabbit
}

National Cancer Institute

\section{Source}

National Cancer Institute. New Zealand White Rabbit. NCI Thesaurus. Code C77112.

An albino New Zealand rabbit with coat genotype c, VV and eye color genotype $\mathrm{np}$. This lagomorph has non-pigmented skin and long slender ears which allows for ease of visualization of peripheral vasculature, thus facilitating phlebotomical procedures. Most common breed utilized in biomedical research and is used in a variety of studies including developmental toxicity studies, toxicology studies, asthma research, and antibody production. 